A N N A L E S Annales de Bretagne et des Pays de l'Ouest

\title{
Les Osismes, peuple de l'occident gaulois
}

Jean-Yves Eveillard

\section{(2) OpenEdition}

\section{Journals}

Édition électronique

URL : http://journals.openedition.org/abpo/3153

DOI : $10.4000 / a b p o .3153$

ISBN : 978-2-7535-4882-4

ISSN : 2108-6443

\section{Éditeur}

Presses universitaires de Rennes

\section{Édition imprimée}

Date de publication : 15 décembre 2015

Pagination : 145-148

ISBN : 978-2-7535-4880-0

ISSN : 0399-0826

\section{Référence électronique}

Jean-Yves Eveillard, «Les Osismes, peuple de l'occident gaulois». Annales de Bretagne et des Pays de l'Ouest [En ligne], 122-4 | 2015, mis en ligne le 15 décembre 2015, consulté le 22 septembre 2020. URL : http://journals.openedition.org/abpo/3153 ; DOI : https://doi.org/10.4000/abpo.3153 


\section{Comptes rendus}

GaLliou, Patrick, Les Osismes, peuple de l'occident gaulois, Coop Breizh, Spézet, 2014, $487 \mathrm{p}$.

Près de quarante ans après la parution de la thèse de Louis Pape, qui nous a quittés en décembre 2014 (L. PAPE, La civitas des Osismes à l'époque gallo-romaine, Librairie Klincksieck, Paris, 1978, 245 p. + 296 p.), Patrick Galliou a livré une monographie renouvelée et augmentée de cet ancien peuple de l'Extrême-Occident. C'est légitimement, peut-on dire, qu'il est revenu aux éditions Coop Breizh, dont le siège est situé dans les limites du territoire osisme, d'assurer la publication de l'ouvrage. Comme chacun sait, les textes des auteurs anciens étant peu prolixes quand il s'agit de ce peuple et aussi des autres peuples armoricains, la rédaction de ce livre nécessitait avant tout une bonne connaissance des découvertes archéologiques. Et dans ce domaine, Patrick Galliou était incontestablement l'homme de la situation : auteur de la Carte archéologique du Finistère (P. Gallou, Carte archéologique de la Gaule. Le Finistère 29, Académie des Inscriptions et Belles-Lettres, Paris, 2010, 495 p.), il a collaboré à celle des Côtes-d'Armor et a dirigé la carte du Morbihan, soit plus que l'ensemble des territoires recouvrant celui des Osismes. Il a participé aux côtés de René Sanquer depuis les années 1970 à de nombreuses campagnes de fouilles : celles des villae gallo-romaines de Keradennec en Saint-Frégant, du Valy-Cloistre en La Roche-Maurice, de Kervennenec en Pont-Croix, pour ne citer que les principales, et dans les années 1990, il a co-dirigé avec Barry Cunliffe de l'université d'Oxford, à qui l'ouvrage est dédié, les importantes fouilles de l'oppidum du Yaudet en Ploulec'h. Ajoutons à cela une connaissance encyclopédique de la bibliographie (elle occupe 51 pages à la fin de l'ouvrage), en particulier de la bibliographie en anglais, connaissance à l'origine de multiples comparaisons éclairantes lorsque la documentation propre aux Osismes fait défaut.

L'ampleur de l'ouvrage (487 pages) tient à la perspective longue dans laquelle l'auteur se place, de la charnière entre le Bronze moyen et le Bronze final (vers 1275 av. J.-C.), à la fin de l'époque romaine (476 après J.-C.), soit près de deux millénaires. Cette option, plutôt inhabituelle, les auteurs se cantonnant le plus souvent dans leur période de prédilection, a le grand avantage de nous faire mieux saisir les continuités et les ruptures dans l'histoire de ce peuple. Le premier chapitre porte sur le nom et les limites du territoire. La forme grecque Osismioi, rapportée par le géographe Strabon à l'occasion du récit du voyage de Pythéas vers 325 av. J.-C., est un ancien Ostimioi comme il nous l'explique, supposant une forme antérieure, non attestée, ${ }^{*}$ postimios. Dès lors le sens est clair : ce sont les "ultimes ", ceux qui habitent le bout du monde connu, sens repris par exemple dans " de fine terre " appliquée à l'abbaye de SaintMathieu, dans le nom moderne Finistère ou dans la forme bretonne Penn ar bed. S'il n'y a pas de doute sur la situation du territoire dans la partie occidentale de la péninsule armoricaine, la question de son étendue exacte et de ses limites orientales est-elle résolue? Patrick Galliou s'en tient à un consensus qui résulte pour les différents peuples armoricains des réflexions de Pierre Merlat et de François Merlet au début des années 1950 : la limite orientale des Osismes traverserait la Bretagne de 
l'embouchure du Gouët à Saint-Brieuc, au nord, à celle de l'Ellé, à Quimperlé, au sud, soit un territoire qui couvre tout le département du Finistère, une partie des Côtesd'Armor comprenant le Trégor et le Goëlo, et écorne même le Morbihan. La répartition des monnaies frappées par ce peuple au temps de son indépendance coïncide avec cette aire géographique. Ainsi défini, le pays des Osismes est de loin le plus vaste de l'Armorique avec $11000 \mathrm{~km}^{2}$ environ. Mais voilà qu'un article récent vient de ranimer la vieille querelle des frontières : selon son auteur (André Cornec), qui argumente à partir de la linguistique bretonne et reprend une hypothèse déjà émise par René Couffon en 1942, la portion littorale des Vénètes ne s'arrêterait pas à l'Ellé mais s'étendrait bien plus à l'ouest jusqu'à l'embouchure du Goyen à Audierne, et de ce point il faut tracer une diagonale jusqu'à Saint-Brieuc, théorie qui ampute considérablement le territoire pris en compte par Patrick Galliou (A. CORNEc, Le territoire des Vénètes et son démembrement, Bull. de la Soc. Arch. du Finistère, t. cXLII, 2014, p. 63-81).

Vient ensuite un précieux chapitre liminaire consacré à la géographie ("Le cadre géographique ") qui fait le point sur la question complexe des fluctuations du niveau marin (p. 28-29), question essentielle quand il s'agit d'un peuple dont le territoire est bordé par la mer sur trois de ses côtés et quand on parcourt son histoire sur une période aussi longue. Puis, Patrick Galliou étudie, le plus logiquement du monde, chapitre par chapitre, l'histoire des Osismes selon l'ordre chronologique : le " Bel âge du bronze ", le premier âge du Fer, le second âge du Fer, l'époque romaine. On ne peut que remarquer un déséquilibre nettement favorable aux deux dernières périodes (91 pages pour le premier âge du Fer, 201 pages pour l'époque romaine, pour 32 pages seulement pour l'âge du Bronze et 31 pages pour le premier âge du Fer) pouvant résulter d'une documentation archéologique moins abondante (?) pour les périodes les plus anciennes, - il est vrai que les découvertes pour l'époque romaine sont nombreuses et d'une plus grande diversité quant à la nature des mobiliers -, mais davantage des spécialités de l'auteur. Néanmoins, même pour les périodes les moins approfondies, l'ouvrage a le grand mérite de nous offrir des synthèses inédites, prenant en compte les avancées les plus récentes de la recherche à l'intérieur du territoire défini, et, à titre comparatif, dans le reste de la Gaule ou en Grande-Bretagne.

Sans trop entrer dans les détails, notons qu'une certaine originalité des populations osismes apparaît dès le premier âge du Fer, mais surtout au second âge (milieu du premier millénaire av. J.-C.) avec, par exemple, l'apparition des souterrains, cavités creusées dans le sol, liées à la présence d'habitats et destinées au stockage des denrées, ou la multiplication des stèles funéraires dont il subsiste un grand nombre. Mais remarquons pour celles-ci qu'il ne s'agit pas uniquement d'une particularité osisme, puisqu'elles sont également abondantes en territoire vénète (carte p. 178). Patrick Galliou ne pouvait passer sous silence la question des mouvements de population et des "migrations celtes " (p. 97-100) : à l'opposé des hypothèses " invasionnistes ", selon lesquelles l'Armorique avec les autres régions tempérées aurait subi l'assaut de populations venues de l'est et parlant celtique, aux alentours des $\mathrm{Vl}^{\mathrm{e}}$ et $\mathrm{V}^{\mathrm{e}}$ siècles av. J.-C., il constate une grande stabilité du peuplement autochtone. Cette constatation irait plutôt dans le sens de l'hypothèse récemment émise par B. Cunliffe et J.-T. Koch que l'auteur développe, hypothèse selon laquelle la langue celtique ne s'est pas diffusée d'est en ouest comme on le disait jusqu'ici, mais serait " née " dans le royaume de Tartessos (sud du Portugal), se diffusant ensuite le long de la façade atlantique avant de gagner l'intérieur du continent (B. CunLIFFE, J.T. Koch [éd.], Celtic from the West. Alternatives Perspectives from Archaeology, Genetics, Language and Literature, Oxford, 2012).

On ne sait rien d'un possible Etat osisme ni de son gouvernement dans les derniers siècles précédant la conquête romaine. Existait-il un " sénat " comparable à celui de leurs voisins les Vénètes que César mentionne à l'occasion de sa campagne 
en 56 av. J.-C.? L'auteur, arguant du fait qu'on ne connait pas d'oppidum central qui eût servi de " capitale ", pense " qu'avant le début du $\mathrm{I}^{\mathrm{er}}$ siècle av. J.-C. (?), cette région occidentale était occupée par des entités politiques autonomes ou même indépendantes " (p. 185). La découverte de résidences aristocratiques comme celle de Saint-Symphorien en Paule, où le culte des ancêtres est attesté par les désormais célèbres bustes en pierre qu'on y a récupérés, permet seulement de dire qu'il existait une élite guerrière sur le territoire (p. 186).

La véritable rupture dans cette histoire longue se situe avec la conquête romaine en 51 av. J.-C. Si les territoires déjà existants avec leurs limites furent conservés, chaque peuple autrefois indépendant forma une civitas, une cité, unité administrative dotée d'une ville chef-lieu nouvellement créée. Pour les Osismes, celle-ci est Vorgium/Carhaix, dans une position remarquablement centrale par rapport aux frontières proposées au début de l'ouvrage. Mais on ne constate pas, à la différence de beaucoup d'exemples dans le reste de la Gaule, de transfert de fonction depuis un oppidum principal vers la ville émergente. Les sites fortifiés au second âge du Fer du camp d'Artus à Huelgoat et de Saint-Symphorien à Paule, bien que peu éloignés de Carhaix, ne semblent pas avoir tenu un tel rôle. Le vaste territoire des Osismes était certainement subdivisé en unités plus petites, les pagi, comme ceux que l'on connaît dans une autre cité armoricaine, celle des Riedones, grâce à plusieurs inscriptions gravées dans la pierre. C'est ici l'occasion de déplorer la pénurie pour le territoire concerné, à quelques rares exceptions près, - les inscriptions qui ont existé étant disparues - de la documentation épigraphique qui nous apprend habituellement tant de choses. On en prend parfaitement conscience à l'examen de l'une de ces inscriptions, celle du Ris à Douarnenez : elle complète l'archéologie sur une activité originale chez les Osismes pour cette époque, celles des salaisons de poissons et de la fabrication du garum, activité placée sous la protection d'une divinité d'origine hellénique, Neptune Hippius, et introduite vraisemblablement par des Méditerranéens. C'est l'un des rares exemples connus d'apport de population " romaine " en Armorique, apport que le public tend généralement à exagérer.

Si la romanisation a entraîné des changements patents dans la manière de vivre des Osismes (urbanisme, techniques de construction, goût du confort, modes vestimentaires, habitudes culinaires, religion, etc.), l'auteur souligne à l'inverse une plus grande continuité dans le monde rural depuis l'âge du Bronze, du moins dans la densité du peuplement et dans les productions agricoles. Mais la principale constante tout au long de ces presque deux millénaires de l'histoire des Osismes - constante qui est en même temps à la source des mutations - tient à la présence de la mer et à la route qui l'emprunte : "Loin d'être un cul-de-sac, le pays osisme fut de la sorte, lors des différentes phases de son histoire, parfaitement intégré aux grands courants culturels et technologiques qui irriguèrent la façade atlantique de l'Europe " (p. 5).

Cette thèse, Patrick Galliou la démontre avec brio, au fil des pages d'un ouvrage foisonnant. Il excelle dans les exercices de synthèse auxquels il nous a déjà habitués et brasse avec une aisance déconcertante une myriade d'informations emmagasinées au fil des années de sa carrière de chercheur. Le livre fourmille d'énumérations qui étayent le propos, de renvois à des passages antérieurs ou postérieurs, de références bibliographiques, au point d'agacer le lecteur non averti. Certes, une telle somme ne saurait être exempte d'imperfections et d'erreurs qui ne seront sans doute relevées que par les spécialistes : ainsi, lorsqu'il rattache les enclos curvilignes au second âge du Fer, les enclos quadrangulaires étant selon lui particuliers à l'époque romaine (p. 109), l'exemple de la ferme gauloise de Rosquelfen en Lanniscat (Côtes-d'Armor) vient contredire cette assertion; à l'occasion d'une référence à des prêtresses qui officiaient dans l'île de Sein, les Gallizenae (p. 174), information rapportée par l'auteur latin Pomponius Mela, c'est à tort que le rapprochement est fait avec un passage de 
Strabon qui concerne Déméter et Coré... Mais de notre point de vue, la principale faiblesse du livre tient aux illustrations. Elles sont nombreuses mais trop souvent de qualité insuffisante. Les cartes de synthèse, si utiles, sont pour nombre d'entre elles trop petites et difficilement lisibles, ce que nous a fait remarquer un lecteur. Trop de photos en noir et blanc sont sombres et inexploitables (p. 24, 27, 40, 86, 118, 119, 167 etc.), défaut dû au tirage et sans doute à la qualité du papier. On aurait souhaité que les illustrations en couleur fussent plus nombreuses et les œuvres d'art du pays osisme, en particulier les sculptures, mieux représentées. Un lexique expliquant certains termes techniques (opus quadratum, opus mixtum, limitanei, origo, comitatenses...) inconnus du grand public aurait été fort utile. Il est dommage que ces faiblesses viennent un peu entacher l'image d'un livre qui est d'une si grande richesse. À ce jour, aucun autre peuple de l'ancienne Armorique, ni même aucun peuple dans l'ensemble de la Gaule, ne bénéficie d'une monographie aussi complète.

Jean-Yves EvEILLARD

Dalarun, Jacques et Le Hü̈rou, Armelle (dir. et trad.), Claire d'Assise. Écrits, Vies, Documents, préface d'André Vauchez, introduction de Maria Pia Alberzoni, Marco Bartoli, Alfonso Marini, Paris, Les éditions du Cerf/Les Éditions Franciscaines, coll. « Sources franciscaines ", 2013, 1098 p.

En 2010, à l'occasion du vIII ${ }^{\mathrm{e}}$ centenaire de la naissance du mouvement franciscain, la collection "Sources franciscaines " publiait la traduction française des sources concernant saint François d'Assise. Trois ans après seulement, c'est à Claire d'Assise, son âme sœur fondatrice de l'Ordre des pauvres dames, qu'est consacré un important volume collectif dirigé par Jacques Dalarun et Armelle Le Huërou sous le titre de Claire d'Assise. Écrits, Vies, Documents. Sur près de 1100 pages distribuées en trois grandes sections, le volume renferme quelque 92 documents qui justifient qu'on puisse désormais le considérer comme le Totum clarien, pendant du Totum franciscain paru en 2010.

Une première partie est baptisée sobrement "Claire d'Assise, écrits ». On sait que Claire d'Assise fut une pionnière dans la mesure où elle fut la première femme à composer une réglementation de la vie religieuse destinée à d'autres femmes, ses sœurs, une "forme de vie de l'ordre des sœurs pauvres " (même l'exceptionnelle Héloïse avait demandé à Abélard de rédiger la règle du Paraclet, sans s'y essayer elle-même). Outre cette Règle, elle laissa aussi son Testament, des Lettres, une Bénédiction et une prière, autant de textes rassemblés ici et qui formaient jusqu'alors la collection " canonique " des écrits de Claire : or cette collection se trouve augmentée d'un texte jusqu'alors cantonné dans le groupe des documents divers bien que riche d'enseignements, un acte de 1238 qui permet de mieux comprendre comment fut mis en œuvre le choix de la pauvreté dans la première communauté : comme l'affirme Jacques Dalarun, sans nul doute, ce texte aussi est bien de la première abbesse de Saint-Damien. Un des premiers mérites de ce recueil et de son organisation est donc de faire apparaître Claire comme un auteur à part entière, et de briser une fois pour toutes avec l'image d'une pâle réplique de saint François au féminin.

Claire mourut en 1253 mais elle fut, de son vivant même, considérée comme sainte. C'est à cette dimension que nous introduit la seconde partie du volume, avec le dossier hagiographique intitulé " Un procès de sainteté ». Le procès de canonisation en constitue bien sûr la pièce maîtresse : outre qu'il fournit sur Claire des témoignages personnels, voire intimes, il retient particulièrement l'attention de tous ceux 